\title{
A new metric to measure success of growth plan
}

\author{
Zhaoyang Lu \\ School of Electrical \& Electronic Engineering, North China Electric Power University, \\ Baoding 071000, China \\ Izy1996ncepu@outlook.com,
}

Keywords: Total Growth, Environmental Growth, Developed Growth Metric.

\begin{abstract}
Total Growth Metric (TGM) to measure success of growth plan, and divide it into Environmental Growth Metric (EGM), Developed Growth Metric (DGM) and Social Growth Metric (SGM) by three E's of sustainability. We consider resource consumption and pollution discharge for EGM. For DGM, not only income level but also life expectancy and education level are considered for comprehensiveness. The SGM is composed of Gini coefficient and corruption perceptions index. After normalizing metrics, we can get the expression of TGM about EGM, DGM and SGM and provide sensitivity analysis to our metrics.
\end{abstract}

\section{Introduction}

The world is rapidly urbanizing, according to United Nations, urban population will account for $66 \%$ of total population by 2050. In order to deal with rapid urbanization, Smart Growth has been raised as an urban planning theory. Smart growth is a way to build cities, towns, and neighborhoods that are economically prosperous, socially equitable, and environmentally sustainable and it has 10 basic principles, such as mix land uses, taking advantage of compact building design, creating walkable neighborhoods and so on.

Our objective is to develop an evaluation system to measure the success of smart growth plan. According to three E's goal, our metric should be consisted with environmental development, economic development and social development. We try to find these factors and provide growth plan for cities based on them.

\section{Overview}

In this section, we establish a metric model to measure the success of smart growth of a city, named Total Growth Metric (TSM). It is an integrated index to measure the effective degree of the smart growth. If the value of TSM is larger, it means the city's smart growth plan is more helpful for sustainable development.

To consider the three E's as well as the demographics, growth needs, and geographical comprehensively, the Total Growth Metric (TSM) can be categorized into environmental growth metric, developmental growth metric and social growth metric. Environmental Growth Metric (EGM) evaluates the success of smart growth from the perspective of environment. To measure the level of development of a city, we have Developed Growth Metric (DGM). In order to assess the economy and development comprehensively, we consider not only people's income level but also other factors which we think are mainly affected by DGM. We will give detailed description in the following section. Similarly, we have Social Growth Metric (SGM) to reflect socially equitable.

In order to unify all metrics to the same value, we carry out the dimensionless processing of these metrics so that we can avoid complex principal component analysis. Finally, we carry on all metrics and obtain a comprehensive metric TGM to measure the success of growth plan. 


\section{Environmental Growth Metric}

We think that the EGM refers to not only pollution discharge, resources consumed during urban development is another important factor. Therefore we divide EGM into two components, Resource Consumption (RC) and Pollution Discharge (PD). And the EGM is the result of the arithmetical average of Resource Consumption (RC) and Pollution Discharge (PD). So we have [1]:

$$
E G M=\frac{1}{2}(R C+P D)
$$

In the formula above, $\mathrm{RC}$ is composed of three indicators: energy consumption, water consumption and land consumption. Also, PD is made up of three indicators: water pollution, air pollution and solid pollution. We think both RC and PD is equal to the arithmetical average of their components, that is

$$
\begin{aligned}
R C & =\frac{1}{3}\left(R C_{E}+R C_{W}+R C_{L}\right) \\
P D & =\frac{1}{3}\left(P D_{W}+P D_{A}+P D_{S}\right)
\end{aligned}
$$

In our model, the value of EGM ranges from 0 to 1 . By formulas above, the increase in the value of RC and PD leads to the larger value of EGM. That is to say, larger value of EGM implies more water, land, energy and materials are consumed and more wastewater, waste gas and waste solid are discharged. Thus, the closer to 1 the EGM is, the more unsuccessful the smart growth is. The closer to 0 , the more effective the smart growth plan is. According to literature, the average value of EGM is 0.44 .

\section{Developed Growth Metric}

There are many factors reflecting the level of economic development, most of which are related to people's income. But evaluating the development level of a city cannot just depend on its economic development, thus we think these factors are far less sufficient to meet our need. We develop DGM taking income level as well as life expectancy and education level into account. We use Income Level (IL) to represent per capita national income and Human Life Expectancy (HLE) to represent life expectancy at birth. And Education Level (EL) is the degree of education.

According to Human Development Report released by United Nations Development Program (UNDP), the DGM is geometric mean of Income Level (IL), Human Life Expectancy (HLE) and Education Level (EL) [1]:

$$
D G M=\sqrt[3]{I L \times H L E \times E L}
$$

- Income level (IL): In the United Nations Human Development Report 2015, the income level is calculated from per capita national income (GNIpc). Besides, to consider the impact of population growth and population aging on the economy, we introduce Population Index (PI) which can be found in relevant literature. [2]Using a logarithmic approach to normalizing IL, we have

$$
I L=\frac{\ln \left(G N I_{p c}\right)-\ln (100)}{\ln (75000)-\ln (100)} \times P I
$$

where the constants 75000 and 100 are the maximum and minimum per capita domestic income.

- Human Life Expectancy (HLE): The human life expectancy is measured by per capita life expectancy (LE). The maximum and minimum life expectancy values are 85 and 20 set by UNDP, so we can standardize the HLE

$$
L E I=\frac{L E-20}{85-20}
$$

- Education Level (EL): We measure the education level from two aspects-school education index (SEI) and expected school education index (ESEI). SEI is measured by per capita 
education years (SE) and ESEI by per capita expected education years (ESE). The constant 0, 15 and 18 are the minimum, maximum and expected maximum years of schooling set for UNDP. Based on this, we can normalize SEI and ESEI.

$$
\begin{aligned}
S E I & =\frac{S E-0}{15-0} \\
E S E I & =\frac{E S E-0}{18-0}
\end{aligned}
$$

And the EI is the geometric average of SEI and ESEI:

$$
E I=\frac{1}{2}(S E I+E S E I)
$$

According to formulas above, we can calculate the value of DGM. The DGM value ranges from 0 to 1 , and the closer to 1 it is, the higher the urban development level is. It means the smart growth plan

\begin{tabular}{|c|c|c|c|c|}
\hline DGM & DGM $<0.55$ & $0.55<$ DGM $<0.7$ & $0.7<$ DGM $<0.8$ & DGM $>0.8$ \\
\hline LEVEL & $\begin{array}{l}\text { Low human } \\
\text { development } \\
\text { level }\end{array}$ & $\begin{array}{l}\text { medium human } \\
\text { development } \\
\text { level }\end{array}$ & $\begin{array}{l}\text { High human } \\
\text { development } \\
\text { level }\end{array}$ & $\begin{array}{c}\text { Extremely high } \\
\text { human } \\
\text { development } \\
\text { level }\end{array}$ \\
\hline
\end{tabular}
is successful. Table 2 shows different levels corresponding to different values.

Table 1 Human development level based on DGM

\section{Social Growth Metric}

To measure social equitable, we define Social Growth Metric (SGM) which is determined by two factors Gini Coefficient (GC) and Corruption Perceptions Index (CPI). The GC is an indicator to determine the fair degree of income distribution defined by the Lorenz curve. [3] It ranges from 0 to 1 , 1 means that the income distribution among residents is absolutely unequal while 0 means that the income distribution among residents is absolute average. The GC is expected to vary from 0.2 to 0.4 .

The CPI generally defines corruption as "the misuse of public power for private benefit." Transparency International has published CPI since 1995 and we use normalized values, so its value also ranges from 0 to 1 . [4]

We believe that the GC and CPI can be combined to reflect a city's fairness and we have

$$
S G M=\sqrt{G C \times(1-C P I)}
$$

Based on what we have discussed above, SGM varies from 0 to 1 . The increasing value of GC or the decreasing value of CPI means a larger SGM. And a larger means a fairer society that we want. The average level of SGM is 0.5 .

\section{Conclusion}

In the above sections, we discussed Environmental Growth Metric (EGM), Developed Growth Metric (DGM) and Social Growth Metric (SGM). We assume that if TGM is larger than 1, the growth plan is successful and larger value of TGM indicates more successful smart growth, so the TGM is proportional to DGM and SGM and inversely proportional to EGM. Detailed information is shown in figure 1.

$$
T G M=\frac{D G M \times S G M}{E G M}
$$




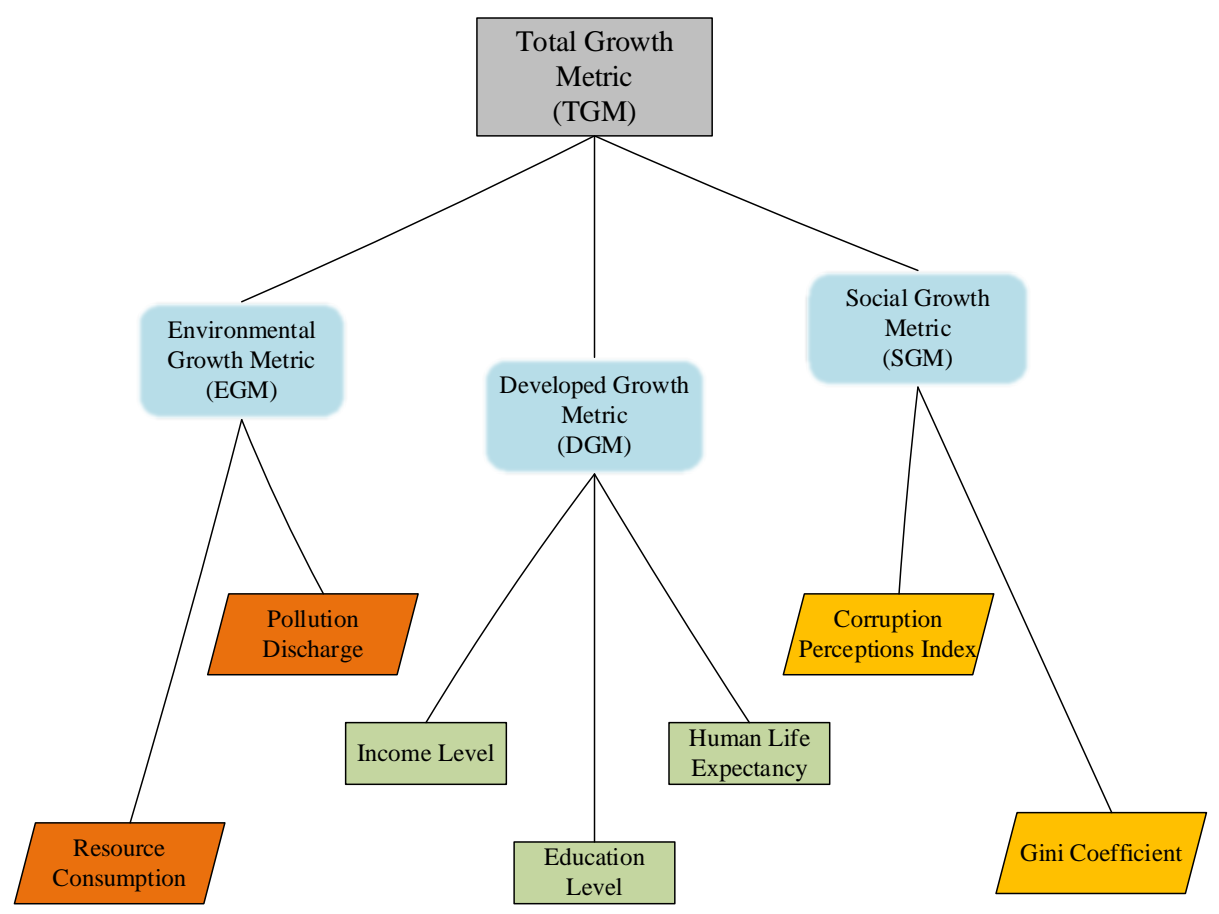

Figure 1 The TGM metric system

\section{References}

[1] UNDP, China Sustainable Cities Report 2016, from http :// www. cn. undp. org/ content /dam /china /docs /Publications / UNDP: page 27-29.

[2]Angang $\mathrm{Hu}$, Shenglong Liu, Zhengguo Ma, Population Aging, Population Growth and Economic Growth: Evidence from China’s Provincial Panel Data: Vol.36, No.3, May 2012, page 17.

[3] Gini Coefficient, from http://baike.baidu.com/

[4] Corruption Perceptions Index ( CPI ), from https: // en. Wiki pedia. org/ wiki/ Corruption_ Perceptions_Index 